\title{
Rac 1 project against diabetes mellitus via attenuation of platelet chemokines
}

\begin{abstract}
Rundk Ahmad Hwaiz ${ }^{1 *}$
\section{Abstract}

Background and Objective: Diabetes mellitus is a major risk factor for platelet activation. Inflammation and abnormal platelet function contribute to morbidity and mortality in diabetes mellitus. So far, the molecular mechanisms involved in the inflammation effects of diabetes are only partially known. Rac1 has been reported to regulate diverse functions in platelets; we hypothesized herein that Rac1 might regulate platelet release of CCL5 and CXCL4 in Diabetes Mellitus.

Methods: We examined the effect of diabetes mellitus on platelet chemokines and Rac1 activation, a small $\mathrm{G}$-protein involved in the activation of platelet. A total of 90 subjects were examined, which included 60 patients with type 2 diabetes and 30 non-diabetic controls. Serum concentrations platelet chemokines (CCL5 and CXCL4) were assayed using ELISA technique. Moreover platelet from C57BL/6 mice were pre treated with a specific Rac1 inhibitor NSC23766 (N6-[2-[[4-(diethylamino)-1-methylbutyl] amino]-6methyl-4-pyrimidinyl]-2 methyl-4, 6-quinolinediamine trihydrochloride), and activated with $25 \mathrm{mmol} /$ /glucose and examined for Rac1 activation by electrophoresis.

Results: Our results showed that platelet chemokines of diabetic individuals and in platelet treated with high glucose is associated with an increased platelet chemokines and Rac1 activation respectively. More important, selective Rac1 inhibition by NSC23766 protected from increased platelet chemokines secretion induced by glucose.

Conclusion: Our study demonstrates that Rac1 plays a crucial role in diabetes-induced inflammation, and it could be a target of novel therapeutic approaches to reduce inflammation in diabetes mellitus. Targeting inflammatory pathways could possibly be a component of the strategies to prevent and control diabetes and related complications.
\end{abstract}

Keywords: Rac1; Platelet; Chemokines; Diabetes Mellitus.

\section{Introduction}

Diabetes mellitus is a serious common chronic, metabolic disease resulting from complex interactions between genetic and environmental factors, including risk factors such as sedentary lifestyle and obesity. ${ }^{1}$ Patients with diabetes have a high risk of developing other health condition like macrovascular and microvascular complications. ${ }^{2}$ Macrovascular and microvascular complications contribute to morbidity and mortality in diabetes mellitus. Previous study showed that mean platelet volume higher in diabetic group than in impaired fasting glucose group. ${ }^{3}$ Diabetic patients are more prone to increase platelet reactivity. High blood glucose contributes to greater platelet reactivity by promoting glycation of platelet proteins. ${ }^{4}$ Insulin which produced by beta cells of the pancreatic islets has a crucial role in allowing glucose in the blood to enter cells, providing them with the energy to function. Diabetic patients usually have low insulin production or insulin resistance..$^{5}$ Insulin deficiency and insulin resistance increase platelet reactivity. Insulin inhibits activation of platelets. Previous studies have showed that diabetic patients with chronic

${ }^{1}$ Department of Clinical Biochemistry, College of Health Sciences, Hawler Medical University, Erbil, Iraq.

* Correspondence: rundk.hwaiz@hmu.edu.krd 
hyperglycemia have also high reactive oxygen species (ROS) production in endothelium as a result of platelet activation. In addition the overproduction of ROS during DM may occur directly through glucose metabolism and auto oxidation or indirectly through the formation of proinflammatory cytokine Receptor for advanced glycation end products (RAGE). ROS playing an important role in the development of vascular damage and enhance the activation of other signaling molecule like, NF-B and protein kinase $C$ (PKC), leading to redox sensitive gene transicription. ${ }^{6}$ An important proinflammatory derivative such as CXCL4 and CCL5, present in the cytosol of platelets, are produce from mRNA and release up on activation of platelets. Upon platelet activation, these proinflammatory derivatives release by platelets. The activation of Platelet during CLP in mice leads to expression of CXCL4 and CCL5, which induces other immune cells to aggregate and induce more inflammation during sepsis. ${ }^{7,8}$ Rac1 is a small GTPase protein which Rac-1, a small $G$ protein, is an important signaling molecule plays key function in diabetes mellitus. ${ }^{9}$ Moreover, Rac1 controls platelet secretion of CXCL4 and CCL5 in sepsis. $7,8,10$ Other studies have identified an essential role Rac1 in lamellipodia formation, phospholipase Cy 2 activation, granule secretion and clot retraction in platelets. $^{10}$ Thus, I asked whether Rac1 might be involved in the secretion of CXCL4 and CCL5 from platelets in DM the present study.

\section{Methods}

\section{Animals}

The experiments were performed using male C57BI/6 mice (20-25 g) in accordance with the legislation on the protection of animals and were approved by the Regional Ethical Committee for Animal Experimentation at Lund University, Sweden. Animals were anesthetized by intraperitoneal (i.p.) administration of 75 mg ketamine hydrochloride (Hoffman-La
Roche, Basel, Switzerland) and $25 \mathrm{mg}$ xylazine (Janssen Pharmaceutica, Beerse, Belgium) per kg body weight. A total of 90 subjects were examined, which included 60 patients with type 2 diabetes and 30 non-diabetic controls in (University Hospitals Malmö) has systematically collected plasma and all consenting DM patients. Patients didn't have drugs affecting platelet for previous 2 weeks. The general inclusion criteria for entering in the study were: male and female, age 18-65 years. The subjects who included in this study were diagnosed DM according to the definition established by the International Diabetes Federation. The criteria were confirmed in order to subject to be diagnosed with DM should have the following conditions: Overweight male or female with waist circumference $\geq 94 \mathrm{~cm}$, and $\geq 80 \mathrm{~cm}$, respectively; Fasting glucose level $\geq 100 \mathrm{mg} / \mathrm{dL} ;$ and, Male HDLcholesterol $<40 \mathrm{mg} / \mathrm{dL}$, female HDLcholesterol $<50 \mathrm{mg} / \mathrm{dL}$. The matched age control group inclusion criteria were: Healthy volunteers were recruited among personal and plasma donors of the center. All of them were normolipidemic (fasting total cholesterol and triglycerides below the 90th percentile of the population), normoglycemic (fasting glucose $<100 \mathrm{mg} /$ $\mathrm{dL})$, and non-obese (BMl $<30 \mathrm{~kg} / \mathrm{m} 2)$.

\section{Plasma samples}

Plasma samples were obtained following the standard procedures: a $4 \mathrm{~mL}$ (EDTA) blood sampling tube wasused for collection of plasma from peripheral venous, (BD vacutainers, Plymouth, UK). The blood sampling tubes were centrifuged $(1300 \times \mathrm{g}$, $10 \mathrm{~min}$ at $4^{\circ} \mathrm{C}$ ) within 1 hour. Plasma was then isolated and immediately frozen in aliquots at $-20^{\circ} \mathrm{C}$. Samples were transferred to $-80^{\circ} \mathrm{C}$ for long-term storage.

\section{ELISA}

Plasma CCL5 and CXL4 levels were successfully measured in 90 individuals assessed by enzyme-linked immunosorbent assay (ELISA) using the Quantikine ELISA kit for Human CCL5/ RANTES (R\&D Systems, Minneapolis, 
USA, cat no. DRN00B) and Human CXCL4 (R\&D Systems, Minneapolis, USA, cat no. AF795) following manufacturer's instructions. Absorbance was measured at $450 \mathrm{~nm}$. Readings at $570 \mathrm{~nm}$ were subtracted from these at $450 \mathrm{~nm}$, to correct for plate imperfections. All samples were assessed in duplicate. On each microplate, a new standard curve was established by diluting a standard with known concentration. Mean absorbance for the duplicate wells was used to determine the chemokines concentration for each sample, using a logistic curve-fitting algorithm. With appropriate dilutions, all absorbance values were within the linear portion of the standard curve. Concentrations read from the standard curve were multiplied by the dilution factor. The results were expressed as $\mathrm{ng} / \mathrm{mL}$.

\section{Platelet isolation and Rac1 activity}

Blood was collected in $1-\mathrm{ml}$ syringes containing $0.1 \mathrm{ml}$ of acid-citrate-dextrose anticoagulant, immediately diluted with equal volumes of modified Tyrode solution (1 $\mathrm{mg} / \mathrm{ml}$ prostaglandin $\mathrm{E} 1$ and $0.1 \mathrm{U} / \mathrm{ml}$ apyrase), and centrifuged at $200 \mathrm{~g}$ for 5 $\mathrm{min}$ at room temperature. Platelet-rich plasma was collected and centrifuged at $800 \mathrm{~g}$ for $15 \mathrm{~min}$ at room temperature, and pellets were resuspended in modified Tyrode solution. After being washed one more time at $10000 \mathrm{~g}$ for $5 \mathrm{~min}$, platelets were resuspended at a count of $0.5 \times 10^{8}$ platelets per tube in Tyrode solution. Platelets from wild-type C57BL/6 mice were treated with 2 different glucose concentrations, mimicking normoglycemia (5 mmol/L) or hyperglycemia $(25 \mathrm{mmol} / \mathrm{L})$ for 30 minutes, with and without NSC23766 $(100 \mathrm{mM})$ at $37^{\circ} \mathrm{C}$. After stimulation, cells were immediately fixedby the addition of $0.5 \%$ paraformaldehyde, samples were centrifuged at $10000 \mathrm{~g}$ for $10 \mathrm{~min}$ at $4^{\circ}$ C.Cells were resuspended in $1000 \mu$ lysis buffer on ice, and then centrifuged at $16000 \mathrm{~g}$ for $15 \mathrm{~min} ; 10 \mu \mathrm{l}$ from each supernatant was removed to measure the protein content using Pierce BCA Protein Assay Reagent (Pierce Biotechnology) and the rest of the volume was used for the pull-down assay. Supernatant containing equal amount of proteins were then diluted with $2 \times$ SDS sample buffer and boiled for $5 \mathrm{~min}$. Proteins were separated using SDS-PAGE (10-12\% gel). After transferring to a nitrocellulose membrane (Bio-Rad, Hercules, CA, USA), blots were blocked with Tris-buffered saline/Tween-20 containing $3 \%$ bovine serum albumin at room temperature for $1 \mathrm{~h}$, followed by incubation with an anti-Rac1 antibody $(1: 1000)$ at $4{ }^{\circ} \mathrm{C}$ overnight. Binding of the antibody was detected using peroxidaseconjugated anti-mouse antibody (1:100 000; Pierce Biotechnology) at room temperature for $2 \mathrm{~h}$ and developed by I $\mathrm{m} \mathrm{mun}$ - St a r W e s t e r n C Chemiluminescence Kit (Bio Rad). Total Rac1 was used as a loading control.

\section{Statistics}

Data were presented as mean values \pm s.e.m. Statistical evaluations were performed by using nonparametric test (Mann-Whitney). $\mathrm{P}<0.05$ was considered significant and $\mathrm{n}$ represents the total number of mice in each group. Statistical analysis was performed by using SigmaPlot 10.0 software (Systat Software, Chicago, IL, USA).

\section{Results \\ NSC23766 Prevents High Glucose- Induced platelet activation by attenuating Rac1 activation}

To evaluate the in vitro effects of NSC23766 on glucose induced platelet activation, isolated platelet from wild-type C57BL/6 mice were treated with 2 different glucose concentrations, mimicking normoglycemia $(5 \mathrm{mmol} / \mathrm{L})$ or hyperglycemia $(25 \mathrm{mmol} / \mathrm{L})$. Isolated platelet exposed to $25 \mathrm{mmol} / \mathrm{L}$ of glucose for 30 minutes showed a significant Rac1 activation compared with platelet with 5 $\mathrm{mmol} / \mathrm{L}$ of glucose (Figure 1A, B). These data confirm the detrimental effects of high glucose levels on activation of Rac1 in platelets. Interestingly, pretreatment with Rac1 inhibitor, NSC23766 (100 mM), was 
able to protect from platelet activation induced by high glucose (Figure 1A, B).

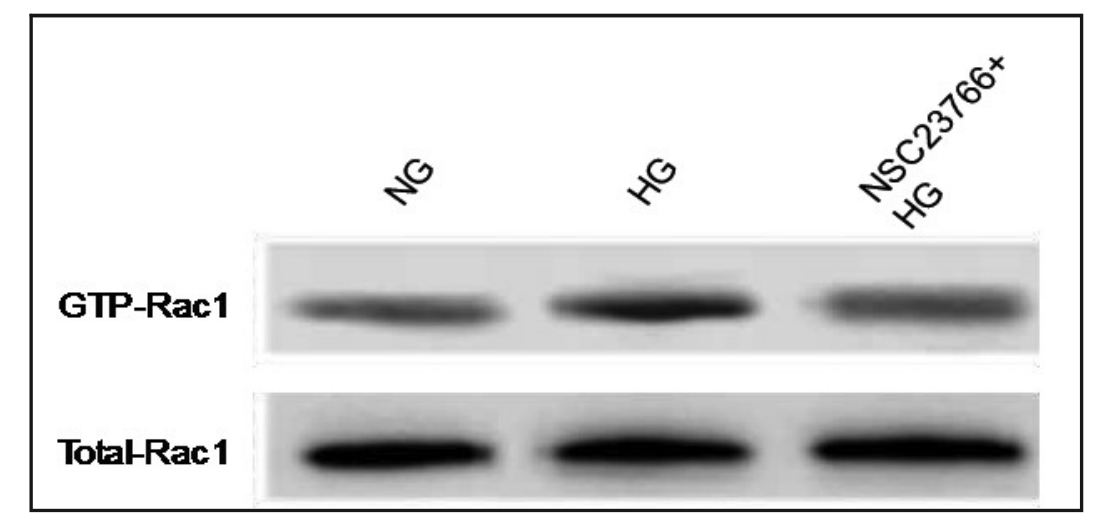

A

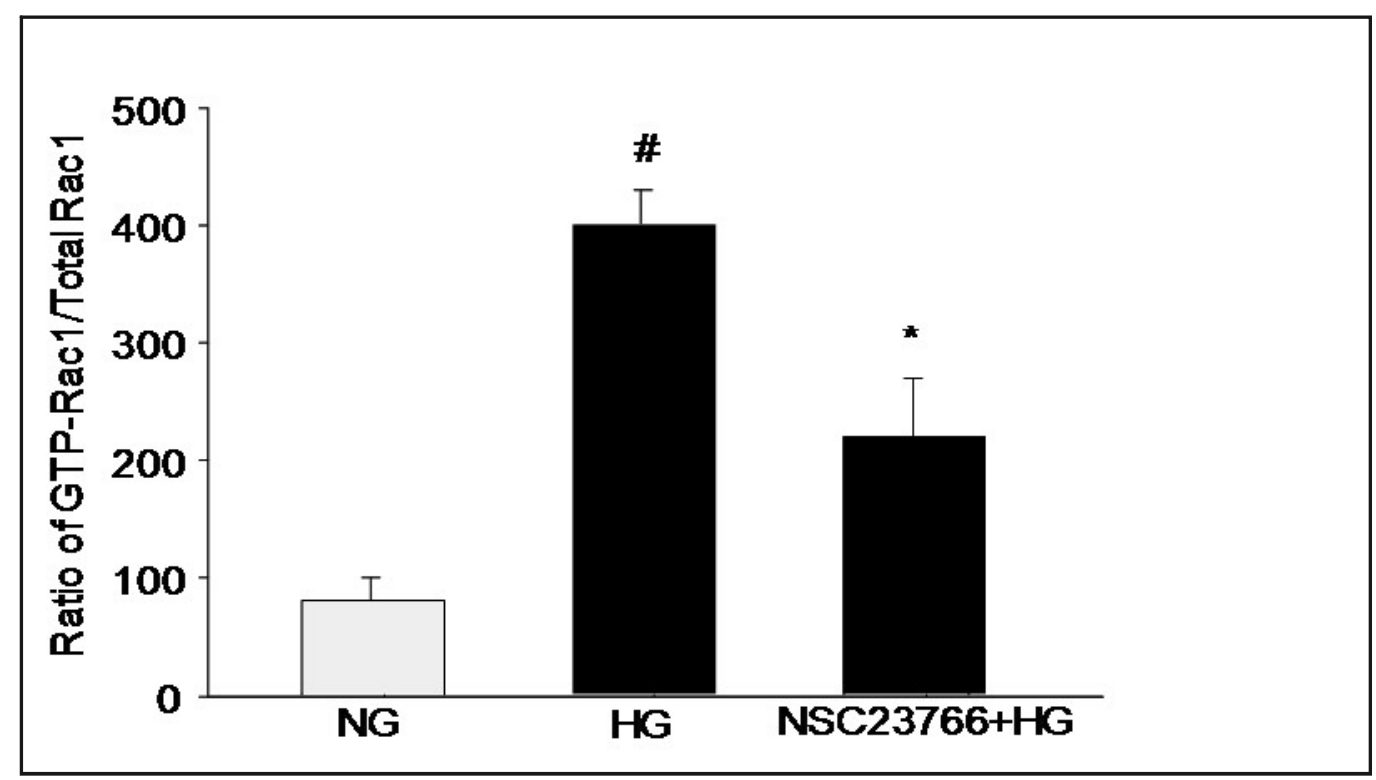

B

Figure1: Rac1 activity in platelet lysate.

A) Rac1-GTP was determined by Western blotting by use of GST-PAK pull-down beads, $30 \mathrm{~min}$ after induction of glucose.

B) Band intensities were quantified in isolated platelets by densitometry and normalized to total Rac1. Western blots are representative of 3 independent experiments. Isolated platelets were treated with the Rac1 inhibitor NSC23766 $(100 \mathrm{mM})$ and induced by different dose of glucose $(\mathrm{NG}=5 \mathrm{mmol} / \mathrm{l})$ or $(\mathrm{HG}=25 \mathrm{mmol} / \mathrm{l}), \mathrm{B}) \mathrm{n}=3, P<0.05$. 
Increased circulating CCL5 and CXCL4 in Diabetes Mellitus

Plasma levels of CCL5 in control group were low but detectable (Figure 2A, $\mathrm{n}=90$ ). Hyperglycemia significantly enhanced plasma levels of CCL5 from 0.6 $\pm 1.1 \mathrm{ng} / \mathrm{mL}$ up to $135.0 \pm 8.2 \mathrm{ng} / \mathrm{mL}$ (Figure 2A, $P<0.05$ versus Ctrl, $\mathrm{n}=90$ ). In addition, I observed that the plasma level of CXCL4 was increased in DM compared with control group from $0.9 \pm 0.2 \mathrm{ng} / \mathrm{mL}$ up to $125 \pm 7.2$ (Figure 2B, $P<0.05$ versus Ctrl, $\mathrm{n}=90)$. Thus, $D M$ significantly induced plasma levels of CCL5 (Figure $2 \mathrm{~A}, \mathrm{~B}$ ).

\section{Discussion}

The present findings indicate an important function of Rac1-mediated activation of platelet and secretion of CXCL4 from platelets in DM. These results point to an important role of platelets in DM and implicate that inhibition of Rac1 signaling and/or CXCL4 function might be useful strategies to ameliorate DM. Platelets are not only critical in wound healing and thrombosis but also exert numerous pro-inflammatory functions in the host response to bacterial invasion. ${ }^{11}$ For example, data have shown that platelets regulate numerous aspects of leukocyte responses to severe infections. ${ }^{7,8,10}$ Previous studies have showed the importance of platelet in DM however, in this study I foundthat the stimulation of isolated platelets by high concentration of glucose induce the activation of Rac1 and interesitingly this increse is abolished by NSC23766. This indicate the importance of platelet and Rac1 in molecular mechanism of DM. Morover the platelet chemokines CCL5 and CXCL4 are found in high concentration of diabetic subjects, demonstraiting the molecular mechanism of platelet in regulating DM. CCL5 and CXCL4 are most abundant chemokines in platelets. ${ }^{7,8}$ Several studies have shown the higher level of soluble inflammatory markers, including interleukin-6 (IL-6), tumor necrosis factor- $\alpha$ (TNFa), CXCL16, and high-sensitivity C-reactive protein (CRP) in patients with metabolic syndrome compared to the healthy subjects. ${ }^{12,13,14}$ In addition, metabolic syndrome has been related to an increased number of several immune cells like leukocyte, monocyte and platelet activation. ${ }^{12,13,14}$ Interestingly, I found that the level of CXL4 and CCL5 in plasma from diabetic patients are higher than in normal subjects (Figure 2A, B). Previous studies showed the importance of Rac1 in regulation of platelet chemokines secretion. ${ }^{7,8,10}$ Morover, it is well established that high glucose level in the blood promote the production of reactive oxygen species (ROS) on endothelial lining of the vessels. ${ }^{15,16}$ ROS is an important
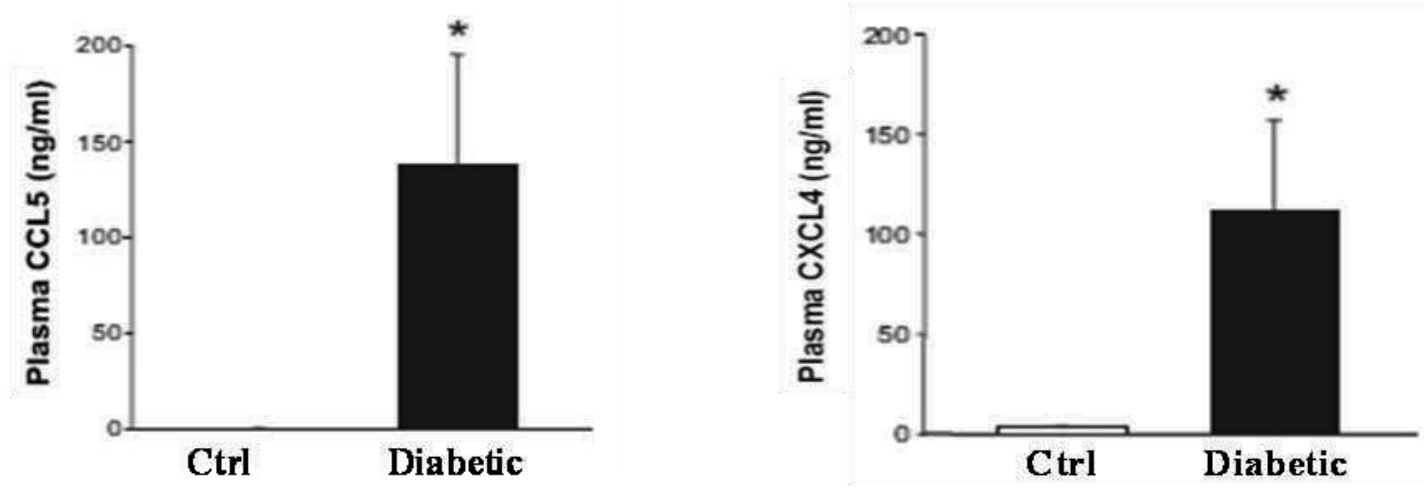

Figure 2: Activated platelets secret chemokines in diabetic patients.

ELISA was used to quantify the levels of CCL5 and B) CXCL 4 in the diabetic patient's plasma. Data represent mean \pm SEM and $\mathrm{n}=90(\mathrm{Ctrl}=30$, Diabetic $=60)$. ${ }^{*} P<0.05$ vs. control. 
signaling molecules which produced by $\mathrm{NADPH}$, play a crucial role in the progression of inflammation and vascular damage in DM. ${ }^{17}$ Rac1, a small G protein, is an important signaling molecule integrating intracellular transduction pathways toward NADPH oxidase activation. ${ }^{18}$ In this study I determined the role of Rac1 in platelet activation. Hence, Rac-1 activation, could be a crucial mechanism for the hyperglycemia-evoked platelet activation, chemokines secretion and vascular oxidative stress in DM. In this study I demonstrated the role of Rac1 in platelet activation as well as the hyper expression of the chemokines CXCL4 and CCL5 as a consequences of platelet activation. Taking together, Rac1 regulate chemokines secretion by activated platelet in DM, Rac1 inhibotor, thus, might be a therapeutic agent to Control DM via attenuation of chemokines which aresignaling molecules in exaggerationg inflammation. However, I need to do more invivo experiment in order to strengthen my hypothesis.

\section{Conclusion}

In this study I demonstrated the role of Rac1 in platelet activation as well as the hyper expression of the chemokines CXCL4 and CCL5 as a consequences of platelet activation. Taking together, Rac1 regulate chemokines secretion by activated platelet in DM, Rac1 inhibotor, thus, might be a therapeutic agent to Control DM via attenuation of chemokines which are signaling molecules in exaggerating inflammation. However, I need to do more invivo experiment in order to strengthen my hypothesis.

\section{References}

1. Liu C, Foti K, Grams ME, Shin JI, Selvin E. Trends in Self-reported Prediabetes and Metformin Use in the USA: NHANES 2005-2014. J Gen Intern Med 2019.

2. Michael J. Fowler, MD. Microvascular and Macrovascular Complications of Diabetes. Clinical Diabetes 2008; 26(2):77-82.

3. Kodiatte TA, Manikyam UK, Rao SB, Jagadish TM, Reddy M, Lingaiah HK, Lakshmaiah V.
Mean platelet volume in type 2 diabetes mellitus. J Lab Physicians 2012; 4(1):5-9.

4. Kumari Shilp, R. M. Potekar. A Study of Platelet Indices in Type 2 Diabetes Mellitus Patients., Indian J Hematol Blood Transfus 2018; 34(1):115 -20 .

5. Balakumar $P$, Maung-U K, Jagadeesh $G$. Prevalence and prevention of cardiovascular disease and diabetes mellitus. Pharmacol Res 2016;113(Pt A):600-9.

6. Daniela Cosentino-Gomes, Nathália RoccoMachado, José Roberto Meyer-Fernandes. Cell Signaling through Protein Kinase C Oxidation and Activation. Int J Mol Sci 2012; 13(9):10697721.

7. Hwaiz R, Rahman M, Zhang E, Thorlacius $H$. Platelet secretion of CXCL4 is Rac1-dependent and regulates neutrophil infiltration and tissue damage in septic lung damage. $\mathrm{Br} \mathrm{J}$ Pharmacol 2015; 172(22):5347-59.

8. Hwaiz R, Rahman M, Syk I, Zhang E, Thorlacius $\mathrm{H}$. Rac1-dependent secretion of platelet-derived CCL5 regulates neutrophil recruitment via activation of alveolar macrophages in septic lung injury. J Leukoc Biol 2015; 97(5):975-84.

9. Schiattarella GG, Carrizzo A, llardi F, Damato A, Ambrosio M, Madonna M, et al. Rac1 Modulates Endothelial Function and Platelet Aggregation in Diabetes Mellitus. J Am Heart Assoc 2018; 7(8).

10. Hwaiz R, Rahman M, Zhang E, Thorlacius H. 2014. Rac1 regulates platelet shedding of CD40L in abdominal sepsis. Lab Invest. Sep;94(9):1054 -63 .

11. Wang Y, Hwaiz R, Luo L, Braun OÖ, Norström $E$, Thorlacius $H$. Rac1 regulates bacterial toxin-induced thrombin generation. Inflamm Res 2018; 65(5):405-13.

12. Mankowska A, Pollak J, Sypniewska G. Association of C-Reactive Protein and Other Markers of Inflammation with Risk of Complications in Diabetic Subjects. EJIFCC 2006; 17(1):8-11.

13. Marques $P$, Collado $A$, Martinez-Hervás $S$, Domingo E, Benito E, Piqueras L, et al. Systemic Inflammation in Metabolic Syndrome: Increased Platelet and Leukocyte Activation, and Key Role of CX3CL1/CX3CR1 and CCL2/CCR2 Axes in Arterial Platelet-Proinflammatory Monocyte Adhesion. J Clin Med 2019; 8(5).

14. Yao L, Herlea-Pana O, Heuser-Baker J, Chen Y1, Barlic-Dicen J. Roles of the chemokine system in development of obesity, insulin resistance, and cardiovascular disease. J Immunol Res 2014; 181450.

15. Volpe $\mathrm{CMO}$, Villar-Delfino $\mathrm{PH}$, Dos Anjos PMF, Nogueira-Machado JA. Cellular death, reactive oxygen species (ROS) and diabetic complications. Cell Death Dis 2018; 9(2):119.

16. Kaneto $H$, Katakami $N$, Matsuhisa $M$, Matsuoka TA. Role of reactive oxygen species in the progression of type 2 diabetes and 
atherosclerosis. Mediators Inflamm 2010; 453892.

17. Henríquez-Olguin C, Knudsen JR, Raun SH1, Li Z, Dalbram E, Treebak JT, et al. Cytosolic ROS production by NADPH oxidase 2 regulates muscle glucose uptake during exercise. Nat Commun 2019; 10(1):4623.

18. Hordijk PL. Regulation of NADPH oxidases: the role of Rac proteins. Circ Res 2006; 98(4):453-62. 